\section{Polyvinyl alcohol/cellulose hydrogel as possible corneal stroma substitute in drug permeation tests}

\section{Erica Zucchetti, ${ }^{1}$ Leonardo Ling, 1} Daniela Monti, 1,2 Patrizia Chetoni, 1,2 Silvia Tampucci,1,2 Susi Burgalassi1,2

1Department of Pharmacy, University of Pisa, Pisa; ${ }^{2}$ Italian inter-university centre for the promotion of the $3 \mathrm{Rs}$ in teaching and research, Pisa, Italy

\begin{abstract}
The permeation studies of active compounds and formulations are necessary to verify the capability of molecules to pass through the physiological barrier of our body. In order to develop a 3D model of the cornea, preparation and characterization of different hydrogels as corneal stroma substitutes were tested.
\end{abstract}

\section{Introduction}

The cornea is composed of five layers, but the most important barrier action against the passage of foreign molecules is performed by i) the epithelium, which is a lipophilic structure composed of 5-6 layers of epithelial cells characterized by tightjunctions; and ii) the stroma which constitute a hydrophilic barrier composed of a collagen network, water, extracellular matrix and few fibroblasts. ${ }^{1}$ The employment of the epithelial model in permeation studies has a good correlation with in vivo only for hydrophilic and moderately lipophilic compounds instead, highly lipophilic substances show a higher permeation coefficient in respect to the entire cornea due to the absence of hydrophilic stroma. Therefore, the presence of stroma in corneal permeation model is essential to obtain permeation data for both hydrophilic and lipophilic compounds similar to original tissue. ${ }^{2}$ By the fact that corneal stroma is composed in major part of collagen and water and in a minimal part by cells, it may be produced artificially developing a hydrogel with the appropriate chemical-physical characteristics.

\section{Materials and Methods}

The hydrogels were prepared using
PVA (polyvinyl alcohol 26-88; Merck) in combination with microcrystalline cellulose (Pharmacel 102; DFE Pharma) (PVA/C) or gelatin (Type A porcine gelatin; ACEF S.p.A., PVA/G) and a thermal cross-linking treatment or chitosan (CHI, Powder-Shrimp $87,6 \%$ Deac.; Marine Chemical) by a process of acidification and alkalinization. The PVA/C and PVA/G were prepared by dissolving the component separately: i) cellulose was dissolved in an aqueous solution of urea and sodium hydroxide, stirred and then stored overnight in the refrigerator then warmed up to room temperature and stirred until the solution was transparent; ii) PVA was dissolved in the same mixture under stirring at $90^{\circ} \mathrm{C}$; iii) gelatin was dissolved in water under stirring at $40^{\circ} \mathrm{C}$. Finally, the dispersions were mixed in different ratios and subjected to 7 or 14 freezing/thawing cycles. ${ }^{3}$ Instead, the $\mathrm{CHI}$ hydrogels were prepared by dissolving chitosan in an acetic solution at $60^{\circ} \mathrm{C}$ under stirring. The obtained product was filtered, poured into a 24 wells-plate and added of sodium hydroxide $0.5 \mathrm{M}$. All the hydrogels were then compressed and washed with water until neutral $\mathrm{pH}$. The first characteristics analyzed of the hydrogel were the percentage of mass loss and water content. The last one was compared to that of the corneal stroma. Following, the wettability characteristics of the hydrogel by water have been determined using the captive bubble technique of contact angle goniometry, where an air bubble is placed in contact with the hydrogel surface meanwhile it was suspended in a clear glass chamber of milli-Q water. 4,5 Successively, permeability to a water flow employing an appropriate apparatus able to accommodate the hydrogel and apply a determinate pressure was evaluated and the permeability coefficient $\mathrm{K}$ was calculated. 6 The apparatus consists of a chamber with two apertures: the upper one for the inlet of pressurized water and the lower one to collect the amount of water permeated through the hydrogel. ${ }^{7}$ Finally, the selected hydrogels were subjected to permeation studies of two molecules for glaucoma
Correspondence: Erica Zucchetti, Department of Pharmacy, University of Pisa, Pisa, Italy

E-mail: erica.zucchetti@phd.unipi.it

Key words: Permeation studies; cornea; stroma equivalent; epithelial tissue; hydrogel.

Disclosures: The authors have nothing to disclose.

Conference presentation: This paper was presented at the Third Centro 3R Annual Meeting - L'era delle 3R: modelli in silico, in vitro e in vivo per promuovere la ricerca traslazionale 30 September - 1 October 2021, Evento online organizzato dal Politecnico di Torino.

Received for publication: 9 July 2021.

Accepted for publication: 7 September 2021.

This work is licensed under a Creative Commons Attribution NonCommercial 4.0 License (CC BY-NC 4.0).

CCopyright: the Author(s), 2021

Licensee PAGEPress, Italy

Biomedical Science and Engineering 2021; 4(s1):203 doi:10.4081/bse.2021.203

treatment (Timolol Maleate, TM; and Magl17b, a newly synthetized compound) with different $\log$. The apparatus consists of a perfusion system able to accommodate the hydrogel with a donor and receiving compartment. Samples of receiving phase were withdrawn at the determined times and analyzed with HPLC.

\section{Results}

The PVA/G showed a mass loss similar to the percentage of gelatin contained (Figure 1), therefore gelatin was not crosslinked with this approach and no further investigations were performed on these hydrogels. Instead, PVA/C and CHI showed a negligible mass loss (0-3.7\%) and a water content very close to the normal hydration levels of the corneal stroma. ${ }^{8}$

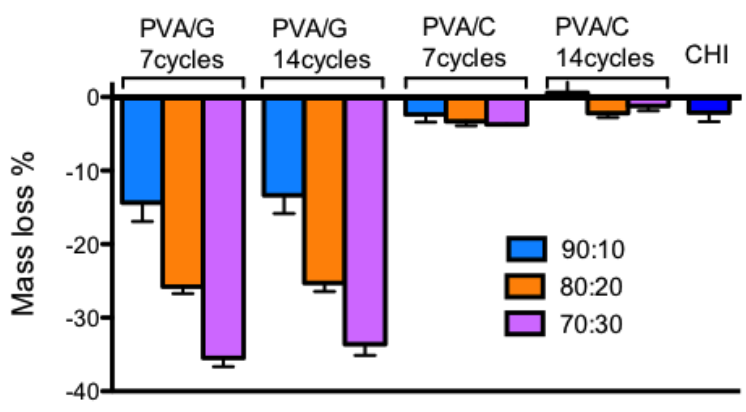

Figure 1. Mass loss percentage 
Table 1. Apparent permeability (Papp) of TM and Magl17b through PVA/C hydrogels and cornea $($ mean $\pm S E)$.

\begin{tabular}{lcc} 
Sulbstrate & Papp TM * $10^{2}(\mathrm{~cm} / \mathrm{h})$ & Papp Magl17b * $10^{2}(\mathrm{~cm} / \mathrm{h})$ \\
PVA/C_90:10 & $5,010 \pm 0,085$ & $0,203 \pm 0,015$ \\
PVA/C_80:20 & $4,767 \pm 0,956$ & $0,210 \pm 0,010$ \\
\hline PVA/C_70:30 & $4,413 \pm 0,230$ & $0,205 \pm 0,005$ \\
Cornea & $1,310 \pm 0,680$ & $0,347 \pm 0,041$ \\
\hline
\end{tabular}

The contact angle measurements for PVA/C showed an increase in hydrophilicity as the amount of cellulose increased moreover, the PVA/C and CHI wettability resulted slightly higher than the native stroma.9-11 The water permeability coefficient of the hydrogels resulted very closer to that corneal stroma at normal hydration level. ${ }^{8}$ Finally, the permeation studies of the two molecules showed (Table 1) as hydrogels were a barrier to Magl17b permeation similar to entire cornea while they were more highly permeated than the cornea by TM. This behavior is due to different partition coefficient of the two drugs which cause different affinity to the hydrophilic membrane.

\section{Conclusions}

PVA/C displayed very similar properties to the corneal stroma even if these materials are at a relatively early stage of research and development. Further studies are needed to progress towards their possible application as functional stroma-like materials, but such promising features deserve to be explored with the study of a larger number of molecules and in combination with the corneal epithelial tissue.

\section{References}

1. Reichl S., Becker U. Cell Culture Models of the Corneal Epithelium and Reconstructed Cornea Equivalents for In Vitro Drug Absorption Studies. In: Ehrhardt C., Kim KJ. (eds) Drug Absorption Studies. Biotechnology: Pharmaceutical Aspects, vol VII. Springer, Boston, MA: 2008.

2. Reichl S, Kölln C, Hahne M, Verstraelen J. In vitro cell culture models to study the corneal drug absorption. Expert Opin Drug Metab Toxicol 2011;7:559-78.

3. Chang C, Lue A, Zhang L. Effects of crosslinking methods on structure and properties of cellulose/PVA hydrogels. Macromol Chem Phys 2008;209:1266.

4. Zhang W, Hallström B. Membrane characterization using the contact angle technique I. methodology of the captive bubble technique. Desalination 1990;79:1.

5. Sanchez I, Carmona FJ, GonzalezPuertas S, et al. Intrasession Repeatability of the Contact Angle Measured Using the Captive Bubble Method and Agreement Assessed Between Different Analysis Software Programs. Eye Contact Lens 2020;46:385-90.

6. Johnson EM, Deen WM. Hydraulic permeability of agarose gels. AIChE J 1996;42:1220.

7. Dillon GP, Yu X, Sridharan A, et al. The influence of physical structure and charge on neurite extension in a 3D hydrogel scaffold. J Biomater Sci Polym Ed 1998;9:1049-69.

8. Refojo MF, Yasuda H. Hydrogels from 2-hydroxyethyl methacrylate and propylene glycol monoacrylate. J Appl Polymer Sci 1965:9;3417.

9. Holly FJ. Biophysical aspects of epithelial adhesion to stroma. Invest Ophthalmol Vis Sci 1978;17:552-7.

10. Gupta S, Goswami S, Sinha A. A combined effect of freeze- thaw cycles and polymer concentration on the structure and mechanical properties of transparent PVA gels. Biomed Mater 2012; 7:015006.

11. Shalom TB, Nevo Y, Leibler D, et al. Cellulose nanocrystals (CNCs) induced crystallization of polyvinyl alcohol (PVA) super performing nanocomposite films. Macromol Biosc 2019;19: 1800347. 